\section{Toward trustworthy, useful and independent guidelines - acknowledging conflicts when they exist}

Dr. Wei-Yi Song's letter to CMAJ, “Don't freeze specialists out of guideline development," outlines the Canadian Psychiatric Association's concerns regarding Dr. Ismail Jatoi and Dr. Sunita Sah's CMAJ commentary about guideline reform. ${ }^{2}$ In Dr. Song's view, "The simplistic conclusion that specialist physicians recommend more interventions and care because they make more money doing so is shockingly unscientific and denigrating ...."1

We think it is worth rereading the commentary, since it explains in part how feefor-service conflicts may arise and points out how difficult it seems to be for physicians or other health care workers to help professions to recognize them. There is ample evidence that physicians who "interact" with the pharmaceutical industry prescribe more expensive drugs, request more new drugs be added to hospital and community formularies, and prescribe less rationally ${ }^{3-5}$ than doctors who shield themselves from such biases. It is well recognized that prescribers who interact with the pharmaceutical industry believe themselves immune to these effects. ${ }^{3}$ The industry spends its marketing dollars wisely from a business perspective. Failure to acknowledge one's own biases is well known to the social sciences and an important basis for the success of advertising. 6,7

Dr. Song contends that "Guidelines developed by nonspecialists and that are based solely on clinical trial data may oversimplify treatment and ignore clinical scenarios that require comprehensive judgment in addition to data, and may be harmful to patients," but did not reference this statement.

Drs. Jatoi and Sah suggested that specialty societies do have a role, but that guideline panels should be diverse. They recommended the Guidelines International Network suggestion that "health care professionals with conflicts of interest serve as external advisers rather than voting members of guideline panels .... Guideline panels should be multidisci- plinary in composition, independent of the governing bodies of specialty societies .... Panels that comprise only specialists may lack sufficient heterogeneity and potential for voicing dissenting opinions. Guideline panels should ideally contain individuals with expertise in epidemiology, biostatistics and clinical trial methodology." ${ }^{1}$

Having critically appraised many specialty guidelines, we think Dr. Song raises a key issue when he asks, "If specialist clinicians are biased, how are the data (from research done by specialist physicians) not biased from the very beginning?" This is an important issue in the interpretation of even the best large randomized trials in fields as diverse as diabetes, cardiovascular disease, nephrology, respirology, intensive care, infectious disease, pain and psychiatry. Surrogate outcomes favoured by specialists to the exclusion of more clinically important outcomes have led us into innumerable "evidence traps" and invalid if not foolish guidelines over the last decades. Methodological problems guarantee that many apparently obvious findings are almost certainly not true. ${ }^{8}$

We support the suggestions made by Drs. Jatoi and Sah because many of the best physicians are losing faith that they can trust existing guideline processes. Clinical experts with conflicts of interest need to take a step back and recognize that the best solutions to diagnostic and therapeutic dilemmas are likely to emerge from application of the most diverse and unconflicted intellects possible. Guidelines have always been intended to leave clinical decisions in the hands of those who are directly responsible for them individual clinicians and their patients.

We were disappointed that CMAJ published Dr. Song's letter without appropriate disclosure of his interactions with several pharmaceutical companies. Information supplied on the 2016 Canadian Network for Mood and Anxiety Treatments (CANMAT) guideline, of which Dr. Song was a panel member, reflected that, like most of the other CANMAT authors, he received honoraria for ad hoc speaking or advising and consulting or research funding from several pharmaceutical companies. ${ }^{9}$

\section{Aaron M. Tejani PharmD}

Pharmacist, researcher, Therapeutics Initiative, Department of Anesthesiology, Pharmacology and Therapeutics, Faculty of Medicine, University of British

Columbia, Vancouver, BC

\section{Thomas L. Perry MD}

Clinical associate professor and internal medicine physician, Therapeutics

Initiative, Department of Anesthesiology, Pharmacology and Therapeutics, Faculty of Medicine, University of British

Columbia, Vancouver, BC

- Cite as: CMAJ 2019 December 16;191: E1387. doi: 10.1503/cmaj.73669

\section{References}

1. Song WY. Don't freeze specialists out of guideline development. CMAJ 2019;191:E1008.

2. Jatoi I, Sah S. Clinical practice guidelines and the overuse of health care services: need for reform. CMAJ 2019;191:E297-8.

3. Fickweiler F, Fickweiler W, Urbach E. Interactions between physicians and the pharmaceutical industry generally and sales representatives specifically and their association with physicians' attitudes and prescribing habits: a systematic review. BMJ Open 2017;7:e016408.

4. Wazana A. Physicians and the pharmaceutical industry: Is a gift ever just a gift? JAMA 2000;283:373-80

5. Spurling GK, Mansfield PR, Montgomery BD, et al. Information from pharmaceutical companies and the quality, quantity, and cost of physicians' prescribing: a systematic review. PLoS Med 2010; 7:e1000352.

6. Pronin E. Perception and misperception of bias in human judgment. Trends Cogn Sci 2007;11:37-43.

7. Dana J, Loewenstein G. A social science perspective on gifts to physicians from industry. JAMA 2003;290:252-5.

8. loannidis JP. Why most published research findings are false. PLoS Med 2005;2:e124.

9. Lam RW, Mclntosh D, Wang J, et al.; CANMAT Depression Work Group. Canadian Network for Mood and Anxiety Treatments (CANMAT) 2016 clinical guidelines for the management of adults with major depressive disorder (Section 1. Disease burden and principles of care). Can J Psychiatry 2016;61:510-23.

Competing interests: Aaron Tejani is paid by Lower Mainland Pharmacy Services (Fraser Health BC) and Therapeutics Initiative (University of British Columbia), has received honoraria from local and provincial clinician groups for presentations and has received honoraria from the Guidelines and Protocols Advisory Committee, Doctors of BC. Thomas Perry is paid by Therapeutics Initiative and was a consultant to United States and Canadian litigation from 2008 to 2018 that alleged improper or fraudulent marketing by 5 major pharmaceutical manufacturers. 